\title{
A Review of Potential Effect of Nanotechnology and Control Drug Delivery System Introduce Into Ocular Drug Delivery System
}

\author{
Madhusudan Das', Ms Ankita Sharma², Mr. Kaushal K. Chandrul ${ }^{3}$ \\ 1Pharmacy Graduate, ${ }^{2}$ Assistant Professor and Researcher, ${ }^{3}$ Faculty of Researcher and Development \\ 1,2,3Pharmacy Department, Mewar University, Chittorgarh, Rajasthan, India
}

\begin{abstract}
How to cite this paper: Madhusudan Das | Ms Ankita Sharma | Mr. Kaushal K. Chandrul "A Review of Potential Effect of Nanotechnology and Control Drug Delivery System Introduce Into Ocular Drug Delivery System" Published in International Journal of Trend in Scientific Research and Development (ijtsrd), ISSN: 24566470, Volume-3 | Issue-4, June 2019, pp.28-34, URL: https://www.ijtsrd.c om/papers/ijtsrd23 563.pdf

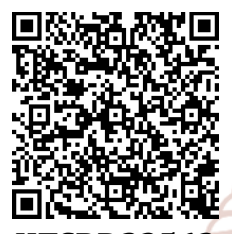
IITSRD23563
\end{abstract}

Copyright (C) 2019 by author(s) and International Journal of Trend in Scientific Research and Development Journal. This is an Open Access article distributed under the terms of the Creative Commons

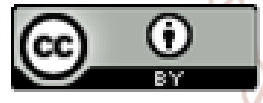
Attribution License (CC BY 4.0) (http://creativecommons.org/licenses/ by/4.0)

\section{INTRODUCTION}

A great approach of nanotechnology is the special treatment for the specific eye disease such as dryness, conjunctiva, eye flu, etc. The main motive of the nanotechnology is the improved the dosage bioavailablity on specific part of eye. Nanotechnology controlled the visuality and protect the eye from the septic situation.[1]

Ordinary ophthalmic measurement shapes are simple to get ready, regulate, and their make taken a toll is relatively moove. watery eye arrangements endure from exceptionally brief contact time with the visual surface and quick nasolachrymal waste, both driving to destitute bioavailability of the sedate. Treatments have visibility and quiet acceptably issues while suspensions frequently donate rise to unusual and variable ocular bioavailability.[2] To address the shortages of ordinary ophthalmic dose shapes, nanotechnology-based frameworks have been examined and a few of which have been created into showcased item. The present chapter outlines the rising part of nanotechnology in ophthalmic sedate conveyance with emphasise what has been licensed over the past decade.

\section{ABSTRACT}

Nanotechnology is a positive concept which will soon improved the field of sedicine and avenues of science. The various types of eye of the medict are expected to be in future. It will not improved our approach to current Nanotechnology is the conducted to special treatment process to minimized the drug adverse effect and their accurate dosage form. The technology is being explore as a means of drug delivery, not only the systemic medication, but also for ocular application as many of the condition.

\section{Keywords: Nanoparticle, liposome, colloidal system, lipid emulsion}

The Anatomy of Eye:

The human eye, exquisite in its detail and plan, speaks to a door to the method we call vision. The eyeball is round in shape and around 1 inch over. It houses numerous structures that work together to encourage locate. The human eye is comprised of layers and inner structures, each of which performs particular capacities. The nitty gritty depiction of each eye portion is given underneath.

\section{A. Sclera.}

The sclera (white parcel of the eye) is the tough white sheath that shapes the outer-layer of the ball. It may be afirm sinewy film that keeps up the shape of the eye as an roughly globe shape. It is much thicker towards the back/posterior viewpoint of the eye than towards the front/anterior of the eye [3]

\section{B. Conjunctiva:}

The conjunctiva may be a lean straightforward mucous epithelial obstruction, lines the interior of the eyelids, and covers the front one-third of the eyeball. The individual portion of conjunctiva is alluded to as the palpebral and bulbar conjunctiva. The conjunctiva is composed of two layers: an outer epithelium and its fundamental stroma 
(substantia propria). The uncovered surface of the eye includes conjunctiva and cornea and is secured with the tear film. The conjunctiva contributes to the arrangement of the tear film by way of emitting significant electrolytes

\section{Cornea.}

The cornea may be a solid clear bulge found at the front of the eye. Surface of the grown-up cornea encompasses a sweep of approximately $8 \mathrm{~mm}$. It has an imperative optical work as it refracts light entering the eye which at that point passes through the student and onto the focal point (which at that point centers the light onto the retina). The cornea, a non-vascular structure (does not contain any blood vessels) gets the essential nutrients from the capillaries that end in circles at its circumference. It is provided by numerous nerves determined from the ciliary nerves.[3] These enter the covered tissue of the cornea. It is in this manner greatly sensitive.

\section{Aquas humour.}

The fluid humor may be a jelly-like substance located within the outer/front chamber of the eye. It could be a watery fluid that fills the "front chamber of the eye" which is located promptly behind the cornea and before the lens. The watery humor is exceptionally marginally antacid salt solution that incorporates minor amounts of sodium and chloride ions. It is ceaselessly delivered, basically by the ciliary processes, streams from the back chamber through the pupil into the front chamber, and exits through the trabecular route at the point and the uveoscleral course. Schlemm's canal (canal of Schlemm or the scleral venous sinus), is a circular channel that collects watery amusingness from the anterior chamber and conveys it into the circulation system by means of the anterior ciliary veins.[4]

\section{E. Pupil.}

Understudy by and large shows up to be the dull "middle" of the eye, but can be more precisely depicted as the circular aperture within the middle of the iris through which light passes into the eye. The size of the student (and thus the amount of light that's conceded into the eye) is directed by the pupillary reflex (too known as the "light reflex").

\section{F. Iris.}

The iris could be a lean circular contractile shade located in front of the focal point but behind the cornea. The iris is a diaphragm of variable estimate whose work is to alter the size of the student to control the sum of light admitted into the eye. It is the coloured portion of the eye (shades may vary separately like blue, green, brown, hazel, or dim)

\section{G. Choroid.}

The choroid layer is found behind the retina and absorbs unused radiation and feeds the external parcels of the retina. It could be a lean, highly vascular (i.e. it contains blood vessels) layer that's dull brown in colour and contains a shade that assimilates abundance light and so anticipates blurred vision (due to as well much light on the retina). The choroid has one of the most elevated blood streams within the body.

\section{H. Vitreous humor.}

The vitreous funniness (moreover known as the vitreous body) is found within the huge region that occupies approximately $80 \%$ of each eye within the human body. The vitreous funniness could be a flawlessly straightforward thin-jelly-like substance that fills the chamber behind the focal point of the eye.[4] It is an albuminous liquid encased in a fragile transparent membrane called the hyaloid layer.

\section{Different mode of ocular drug delivery: Topical route}

Ordinarily topical visual medicate organization is accomplished by eye drops, but they have as it were a short contact time on the eye surface.[5] The contact, and thereby duration of medicate activity, can be delayed by formulation design (e.g.m gels, gelifying details, treatments, and inserts)

\section{Subconjunctival administration}

Customarily subconjunctival infusions have been used to provide drugs at expanded levels to the uvea. Currently this mode of medicate conveyance has picked up new momentum for different reasons. The advance in materials sciences and pharmaceutical definition have given new exciting conceivable outcomes to create controlled release formulations to convey drugs to the back portion and to guide the mending handle after surgery.[5]

\section{Intravitreal administration}

Coordinate sedate organization into the vitreous offers distinct advantage of more direct get to to the vitreous and retina. It ought to be famous; in any case that delivery from the vitreous to the choroid is more complicated due to the prevention by the RPE (Retinal Pigment Epithelium) obstruction. Little particles are able to diffuse quickly within the vitreous but the versatility of large molecules, especially emphatically charged, is limited.

\section{Barrier for ocular drug delivery \\ Drug loss from the ocular surface}

After ingrained, the stream of lacrimal fluid removes ingraining compounds from the surface of eye. Even though the lacrimal turnover rate is as it were around $1 \mu \mathrm{l} / \mathrm{min}$ the excess volume of the ingraining liquid is flown to the nasolacrimal conduit quickly in some of minutes.[5] Another source of non-productive sedate evacuation is its systemic absorption rather than visual retention. Systemic absorption may take put either straightforwardly from the conjunctival sac via local blood capillaries or after the arrangement stream to the nasal cavity.

\section{Lacrimal fluid eye barrier}

Corneal epithelium limits sedate assimilation from the lacrimal liquid into the eye. The corneal epithelial cells form tight intersections that restrain the paracellular medicate permeation. Therefore, lipophilic drugs have ordinarily at slightest an order of greatness higher porousness within the cornea than the hydrophilic drugs.

\section{Blood ocular barrier}

The eye is ensured from the xenobiotics in the blood stream by blood-ocular obstructions. These boundaries have two parts: blood-aqueous boundary and blood-retina barrier. The front blood-eye obstruction is composed of the endothelial cells within the uveam (The center layer of the eye beneath the the sclera.[5] It comprises of the iris, ciliary body, and choroid). This boundary avoids the get to of plasma albumin into the watery humor, conjointly limits the get to of hydrophilic drugs from plasma into the fluid humor. The posterior obstruction between blood stream and eye is comprised of retinal shade epithelium (RPE) and the tight dividers of retinal capillaries. Not at all like retinal capillaries the vasculature of the choroid has broad blood stream and cracked walls. Drugs effortlessly pick up get to to the 
choroidal extravascular space, but there after distribution into the retina is constrained by the RPE and retinal endothelia

\section{Mechanism of ocular drug absorption}

Drugs managed by ingrained must penetrate the eye and do so essentially through the cornea taken after by the noncorneal courses. These non-corneal courses involve drug dissemination over the conjunctiva and sclera and appear to be especially imperative for drugs that are poorly absorbed over the cornea [5].

\section{Various barrier to drug absorption}

In tears have a coordinate bearing on proficiency of drug absorption into the inward eye. The profitable retention of most ophthalmic drugs comes about from diffusional process across corneal membrane. The proficiency of absorption process could be a work of rate and degree at which the transport forms of eye. The flux of any sedate molecule across the natural layer depends on the physicochemical properties of the penetrating particle and its interaction with the film. The degree to which the transport or assimilation prepare happens is additionally work of physiological instrument of precorneal liquid seepage or turnover. In terms of transcorneal medicate penetration, the cornea can be considered to comprise of three essential layers (epithelium, stroma and endothelium). The epithelium and endothelium contain on the order of a 100 overlap more noteworthy sum of lipid fabric than the stroma. [5]Thus, depending on the physicochemical properties of a diffusing medicate, the resistance advertised by the individual layers changes incredibly. Epithelium, being lipodal, represents a diffusional obstruction advertising tall resistance toionic or other watery dissolvable or polar species. In contrast, compounds with generally moo extremity experience a greater diffusional resistance within the hydrophilic stroma layer. This frequently cited concept of sedate penetration over the corneal film ism alluded to as "differential solubility concept

Various factor responsible for dispotion of occuler drug Bioavailability of drugs managed to the eye is an critical thought. There are physiological factors, which can influence a drug's bioavailability counting protein binding, medicate digestion system and lachrymal seepage. Protein bound drugs are unfit of penetrating the corneal epithelium due to the estimate of the protein drug complex. Since of the brief time in which an ophthalmic solution may stay show within the eye (due to lachrymal drainage), protein official of a sedate substance may quickly negate its restorative esteem by rendering it inaccessible for absorption. One of the major issues experienced with conventional ophthalmic arrangements is the quick and extensive elimination of drugs from the precorneal lachrymal liquid. It must be famous that this tall seepage rate is due to the tendency of the eye to preserve its home volume at 7- $10 \mu \mathrm{l}$ for all time, though volumes topically instilled range from 20-50 $\mu \mathrm{L}$. In reality it has been illustrated in vivo that $90 \%$ of the dosage was cleared inside $2 \mathrm{~min}$ for an instilled volume of $50 \mu \mathrm{L}$ and, inside $4 \mathrm{~min}$ for an instilled volume of $10 \mu \mathrm{l}$. Subsequently, the ocular residence time of conventional arrangements is constrained to some minutes, and the overall absIn expansion to the physiological variables affecting ocular bioavailability, other variables as the physicochemical properties of the sedate substance, and item formulation are imperative. Since the cornea could be a membrane-barrier containing both hydrophilic and lipophilic layers, it is permeated most viably by sedate substances having both hydrophilic and lipophilic characteristics. It is advantageous for corneal infiltration to alter the $\mathrm{pH}$ of the solution to increase the extent of unionized medicate within the ingraining dose.

\section{Nasolachrymal drainage system}

The nasolachrymal seepage framework comprises of three parts: the secretory framework, the distributive framework and the excretory framework. The secretory framework comprises of basic secretors that are fortified by squinting and temperature change due to tear vanishing The distributive framework comprises of the eyelids and the tear meniscus around the cover edges of the open eye, which spread tears over the visual surface by squinting, thus preventing dry ranges from creating. The excretory portion of the nasolachrymal seepage framework comprises of: the lachrymal puncta, the prevalent, second rate and common canaliculi; the lachrymal sac and the nasolachrymal conduit. In humans, the two puncta are the openings of the lachrymal canaliculi and are arranged on an hoisted range known as the lachrymal papilla. It is thought that tears are largely absorbed by the mucous layer that lines the conduits and the lachrymal sac as it were a little sum comes to the nasal passage [6] and reflex secretors that have an efferent parasympathetic nerve supply and discharge in response to physical or enthusiastic incitement.

Need to introduced novel drug system in ocular drug delivery:

Ophthalmic medicate conveyance is one of the most interesting and challenging endeavors confronting the pharmaceutical researcher. The scene of ophthalmic drug delivery is profoundly competitive and quickly advancing. New classes of pharmaceuticals and biologics are fueling the demand for novel sedate delivery. The primary point of pharmaco therapeutics is the attainment of compelling sedate concentration at the location of action for the adequate period of time to evoke a response. The challenge is to supply a framework with progressed ocular drug bioavailability and drawn out term of action, but still with a least chance of visual complications. A major problem of ophthalmic sedate conveyance isn't the need of efficient drugs but the fulfillment of their optimal concentration at the location of their ideal concentration at the site of activity $[5,6]$.

The development of unused and inventive implies for improving restorative adequacy proposes that a greater choice of dose shapes will be given to doctors and patients within the following decade. Most of the detailing efforts aim at maximizing visual medicate assimilation through prolongation of the drug home time within the cornea and conjunctival sac, as well as to moderate sedate discharge from the delivery framework and minimize precorneal sedate misfortune. Various ophthalmic definitions and their home time period in the visual depression are given underneath [7]

\section{Formulation Deseign:}

Ophthalmic drugs are defined to bring the active drugs in contact with the eye surface to permit for absorption. Expansion of corneal contact time may result in increased sedate entrance \& higher intraocular drug delivery. In expansion to the dynamic medicate, ophthalmic formulations ought to contain other fixings to control 
various characteristics of the detailing, such as the buffering and $\mathrm{pH}$, osmolality \& tonicity, thickness \& antimicrobial additives. In spite of the fact that these fixings are listed dormant, they can influence porousness of sedate across the visual surface boundaries \& change the therapeutic effectiveness of the sedate.

\section{Controle of ocular infection:}

Visual diseases, both shallow and profound such as conjunctivitis, corneal ulcers and endophthalmitis are caused by differing bunch of microbes, viral and fungal pathogens. Appropriately the armamentarium of available antimicrobials utilized within the anticipation and treatment of these infections incorporates antivirals, antifungals and antibacterials. Common topical antibacterials utilized in treatment of ocular infectious illnesses incorporate sulfonamides, aminoglycosides, polymyxin-based combinations and fluoroquinolones. These fluoroquinolones are demonstrated for extreme bacterial keratitis, endophthalmitis, blepharo-conjunctivitis, corneal ulcers, chronic post-filtration hypotony etc. The fluoroquinolones represent an growing course of wide spectrum antibacterials which cover a have of Gram negative and anaerobic species capable for visual diseases. These antibacterials have picked up notoriety within the ophthalmology field since they have been appeared to be comparable to combination treatment in treatment

Fluoroquinolones offer all the qualities of an ideal antimicrobial operator counting wide antimicrobial spectrum, good tissue entrance and bioavailability, tall rate of clearance, chemical and organic steadiness, moo degree of toxicity, tall authoritative partiality for melanin, superior patient compliance, helpful dose shapes and dosing schedule and moderately moo rate of sedate intuitive.

\section{Mode of Action:}

Fluoroquinolones act by repressing two enzymes involved in bacterial DNA union, both of which are DNA topoisomerases that human cells need which are essential for bacterial DNA replication, subsequently enabling these operators to be both particular and bactericidal. DNA topoisomerases are dependable for isolating the strands of duplex bacterial DNA, embeddings another strand of DNA through the break, and after that resealing the originally separated strands.

DNA gyrase presents negative superhelical twists within the bacterial DNA doublehelix ahead of the replication fork, subsequently catalyzing the division of daughter chromosomes. This action is fundamental for initiation of DNA replication and permits for authoritative of initiation proteins. Topoisomerase IV is mindful for decatenation that's, evacuating the interlinking of daughter chromosomes in this manner permitting isolation into two daughter cells at the conclusion of a circular of replication. Fluoroquinolones connected with the enzyme-bound DNA complex (i.e., DNA gyrase with bacterial DNA or topoisomerase IV with bacterial DNA) to create conformational changes that result within the restraint of normal protein movement. As a result, the modern drug- enzyme-DNA complex blocks movement of the replication fork, subsequently inhibiting normal bacterial DNA union and eventually coming about in rapid bacterial cell passing. More seasoned fluoroquinolones show a relatively steady design with regard to specificity of enzyme restraint in several sorts of microscopic organisms. The newer fourth era fluoroquinolones like moxifloxacin, gatifloxacin have a dual-binding instrument of action, inhibiting both DNA gyrase and topoisomerase IV, in Grampositive species [8,9].

\section{Types of Nanotechnology for used in ocular drug delivery: \\ Types of lipid emulsion:}

LEs are biphasic framework of immiscible fluids (either w/o or o/w types) and one related with soundness issues happening due to aggregation and coalescence of the globules stacking to phase separation [10]. LEs are thermodynamically steady and colloidal. Ls cattering, stabilized by interfacial film of emulsifier. In ocular drug conveyance, they are related with a few points of interest which include sterilization, tall clarity, and ease of arrangement [11].

\section{Oil in water (o/w) lipid emulsion:}

These sorts of emulsion are arranged by utilizing of oil droplet surrounded by surfactant film. The fluid stage is employments as persistent stage, in which oil beads are dispersed. In this type of emulsions, drugs are joined in oil stage and optimized emulsion in best condition [12]. It offers different points of interest which include a straightforward visual sedate conveyance framework, higher stability, sustained impact, and medicate conveyance to more profound layers of the eye and aqueous humor. Diverse drugs like azithromycin, difluprednate etc has been conveyed by this sort of LEs.

\section{Water in oil (w/o) lipid emulsion:}

These sorts of emulsion are for the most part utilized for the hydrophilic drugs. The most points of interest related with $\mathrm{w} / \mathrm{o}$ sort emulsion is spontaneous arrangement and thermodynamically soundness [13]. It has been utilized for the conveyance of certain drugs like dexamethasone, tobramycin [14], chloramphenicol [15]

\section{Bicontineous lipid emulsion:}

In this sort of emulsion, oil and water both exist in continues phase so called as bicontinuous emulsion. The rheological property exhibits non-newtonian stream. The characteristic property is water and oil particle are interlaced which see like crisscross structure [16]. A characteristic property of bicontinous emulsion is that the aqueous and oil nanodomain are isolated by surfactant monolayer [17]. A bicontinous emulsion silicone oil was created and characterized for the diverse parameters to appear its applicability as the cleansing specialists.

\section{Liposome:}

Liposomes are phospholipid bilayer vesicles for focusing on the drugs to the particular location within the body [18]. They give controlled and specific sedate conveyance through upgraded BA [19] and their potential in visual sedate conveyance appears more for lipophilic than. hydrophilic compounds [20]. Liposomes have extra benefit of being completely biodegradable and by and large nontoxic, but stability is exceptionally destitute than particulate polymeric medicate conveyance system. Liposomes are developing devices for sedate conveyance for administration of number of drugs to the eye [21] such as ibuprofen [22], flurbiprofen [23].

Hathout et al. (2007) detailed acetazolamide liposome appeared the emphatically charged and neutral liposomes shown more prominent bringing down in IOP and a more prolonged impact than the adversely charged ones. It was 
suggested that liposomes have improved corneal penetration of medicate by being adsorbed onto the corneal surface, with direct exchange of sedate from liposomes to epithelial cell membranes. Sedate stacking capacity and entanglement efficiency of liposomes depends on numerous components such as measure of liposomes, concentration and sorts of lipid utilized, and physicochemical properties of restorative operator itself. The loading capacity and capture proficiency was moo for Small unilamellar vesicles (SUV) in comparison to Multivesicular vesicles (MLV). In any case, Expansive unilamellar vesicles (LUV) give a adjust between measure, loading capacity and capture proficiency (Ding et al., 2005; Jesorka \& Orwar, 2008).

\section{Colloidal system:}

Visual sedate conveyance is one of the foremost curiously and challenging endeavors confronting pharmaceutical scientists because of the basic and pharmacokinetically specific environment that exists within the eye. The life structures, physiology and natural chemistry of the eye give a special structure that restricts the section of sedate particles at the specified location of action and impenetrable to remote substances. A suitable ocular detailing ought to discharge the medicate overcoming the protective boundaries of the eye without causing permanent tissue harm (Ghate \& Edelhauser, 2006; Urtti, 2006). There are numerous courses of visual organization like topical instillation and subconjunctival infusion for front portion, while periocular and intravitreal infusions, and topical dosing for back fragment. [24]

A fastidious see toward the past of visual delivery clearly emphasizes eye drops as the central and most frequently utilized definition for visual conveyance. In spite of the fact that eye drops are simple to fabricate and quiet compliant but their application is seriously full with issues related to their poor bioavailability (1-10\%). The destitute bioavailability of eye drop may be due to credited restricted range of retention

\section{Microemulsion:}

A picky see toward the past of visual conveyance clearly emphasizes eye drops as the central and most habitually utilized definition for visual movement. In show disdain toward of the truth that eye drops are basic to manufacture and calm compliant but their application is genuinely full with issues related to their destitute bioavailability (1-10\%). The dejected bioavailability of eye drop may be due to credited limited extend of maintenance. MEs have pulled in a extraordinary intrigued as DDS for topical ocular application since of little measure, basic and cheap arrangement, can be sterilized effortlessly by filtration (Soukharev et al., 2005), moo thickness, more prominent capacity as drug carrier, retention promoter, and moo surface pressure of MEs, show spreading on the cornea blending with the precorneal film constituents (Fialho \& da Silva-Cunha, 2004). MEs as carriers for drugs to transport lipophilic and hydrophilic substances through their individual medium, i.e. an watery medium and lipoid medium, and thus move forward the bioavailability (Ma et al., 2008; Kesavan et al., 2013). The infiltration upgrading property of surfactant and co-surfactant of MEs increases the medicate penetrability (sedate take-up) and encourages the passage of sedate by corneal film.

\section{Nanoparticle:}

NPs are one of the most-studied colloidal frameworks with the object of moving forward focusing on of medicate to organs and increasing drug bioavailability over biologic films. NPs are submicroscopic, colloidal framework comprising of macromolecular substances that change in measure from 10 to $1000 \mathrm{~nm}$. In NPs, the drug may be broken down, entangled, adsorbed, joined or encapsulated into the polymer framework. Depending on the method of arrangement, it can be classified into two bunches: NPs (nanospheres) and nanocapsules and have distinctive release profile of medicate (Sahoo et al., 2003; Vandervoor et al., 2007). Nanospheres are little strong circles constituting of dense solid polymeric organize having huge surface region. Drugs can either be joined within the network framework or adsorbed on the surface of the nanospheres. On the other hand, nanocapsules have little central depth (sleek bead) encompassed by a polymeric film. NPs speak to promising drug carriers for ophthalmic applications. After optimal binding to these particles, the sedate retention within the eye is enhanced altogether in comparison to eye drop solutions owing to the much slower visual disposal rate of particles. Smaller particles are superior endured by the patients than larger particles therefore NPs may be an awfully comfortably to be utilized for drawn out activity ophthalmic DDS. Different polymers can be utilized to manufacture NPs such as biodegradable polymers like polylactides, poly (D,L-lactides).

\section{Mode of action of control drug delivery: Diffusion}

Within the Dissemination component, the sedate is released continuously at a controlled rate through the membrane into the tear liquid. In the event that the embed is formed of a strong non-erodible body with pores and scattered medicate. The discharge of sedate can take place by means of dissemination through the pores. Controlled release can be encourage controlled by gradual dissolution of strong scattered sedate inside this matrix as a result of internal dissemination of aqueous solutions. In a solvent gadget, genuine disintegration.

In swelling-controlled gadgets, the dynamic operator is homogeneously scattered in a smooth polymer. Since shiny polymers are basically drug impermeable, no dissemination through the dry matrix occurs. When the embed is put within the eye, water from the tear liquid starts to enter the matrix, at that point swelling and subsequently polymer chain unwinding and sedate dissemination take place. The disintegration of the network, which takes after the swelling handle, depends on polymer structure: linear shapeless polymers break up much faster than cross-linked or somewhat crystalline polymers. Discharge from these gadgets takes after in general Fickian 'square root of time' energy; in some occurrences, in any case, known as case II transport, zero arrange energy has been watched.

\section{Osmosis:}

Within the Osmosis instrument, the embed comprises a transverse impermeable versatile membrane dividing the insides of the embed into a first compartment and a moment compartment; the first compartment is bounded by a semipermeable membrane and the impermeable elastic membrane, and the moment compartment is bounded by an impermeable material and the elastic film. There's a sedate release aperture within the impermeable divider of the insert. The to begin with compartment contains a solute which cannot pass through the semi-permeable [24]. layer and the moment compartment provides a store for the 
sedate which once more is in fluid or gel form. When the embed is put within the aqueous environment of the eye, water diffuses into the first compartment and extends the elastic membrane to extend the primary compartment and contract the moment compartment so that the drug is constrained through the sedate discharge gap.

\section{Bioerosion:}

Within the Bioerosion component, the configuration of the body of the embed is constituted from a matrix of bioerodible fabric in which the drug is scattered. Contact of the embed with tear fluid results in controlled supported discharge of the drug by bioerosion of the framework. The medicate may be dispersed consistently all through the lattice but itis accepted a more controlled discharge is obtained if the sedate is externally concentrated in the matrix. In really erodible or E-type gadgets, the rate of sedate discharge is controlled by a chemical or enzymatic hydrolytic response that leads to polymer solubilization, or corruption to smaller, water-soluble atoms. These polymers, as specified by Heller, [24] may experience bulk or surface hydrolysis. Erodible embeds undergoing surface hydrolysis can show zero arrange release kinetics; given that the gadgets keep up a constant surface geometry which the medicate is poorly water-soluble

\section{Discussion:}

Modern ophthalmic conveyance framework incorporates ocular inserts, collagen shields, visual movies, expendable contact lens and other Novel sedate conveyance frameworks like hiosomes 20 and nanoparticles. More current drift could be a combination of drug delivery innovations for moving forward the therapeutic response of a non useful sedate. This will donate a superior dosage shapes for topical ophthalmic application. Among these medicate conveyance frameworks, as it were few items have been, commercialized. An perfect framework ought to have successful medicate, concentration at the target tissue for a tended period of time with least systemic impact. Understanding acknowledgment is very important for the plan of any comfortable ophthalmic drug conveyance framework. Major Enhancements are required in each framework like advancement in maintained sedate release, large scale fabricating and steadiness. Combination of drug conveyance frameworks might open a modern order for improving the restorative reaction of a non-efficacious system. They can overcome the restrictions and combine the advantages of distinctive frameworks.

NPs are one of the most-studied colloidal frameworks with the object of moving forward

\section{Declaration:}

The article was checked by the authorities of researcher and development (Mewar University) and they not found any inconvenience issue.

\section{References:}

[1] Zarbin MA, Montemagno, Leary JF, Ritch R Nanotechnology in ophthalmology, Can ophthalmol.2010 oct.45[5];457-76

[2] Burke P. Nanotechnology.2005. spring. Graduate class of tought at university. of California, Irvine.
[3] Urtti A. Challenge and obstacle of ocular pharmacokinetics and drug delivery. Adv drug delivery rev, 58, 2006, 1131-35

[4] Jtirvinena K, Tomi J, Urttia SA. ocular absoption following topical delivery. Adv. drug dev, 16, 1995, 319

[5] Meqi SA, Deshpande SG. Ocular drug delivery; control and novel drug delivery. New delhi ;CBS publishers;2002;82-84

[6] Eva M, Amo D, Urtti A, current and future ophthalmic drug delivery system. A shift to the posterior segment drug discov to day;2004,135-45

[7] Blondeau JM, fluroquinolones, Mechanism of action, classification and development of resistances. Surv ophthalmol, $49 ; 2004 ; 73-78$

[8] Desai PN, synthesis and characterization of polyionic hydrogela, Bechalor of homeopathic medicine ana surgery, LMF, homeopathic medical college, india.

[9] He C, Kim SW, Lee DS. insetu gelling stimuli-senstive block copolymer hydrogels for drug delivery. J control release, 127,2008, 189-207.

[10] Pera-Titus M, Leclercq L, Clacens JM, De Campo F, Nardello-Rataj V. Pickeringinterfacial catalysis for biphasic systems: from emulsion design to greenreactions. Angew Chem Int Ed 2015;54:2006-21.

[11] Madni A, Rahem MA, Tahir N, Sarfraz M, Jabar A, Rehman M, et al. Noninvasivestrategies for targeting the posterior segment of eye. Int J Pharm2017;530:326-45.

[12] Daull P, Lallemand F, Garrigue JS. Benefits of cetalkonium chloride cationic oilin-water nanoemulsions for topical ophthalmic drug delivery. J PharmPharmacol 2014; 66:531-41.

[13] Lallemand F, Garrigue JS, Philips B, inventors; Santen Sas, assignee. Water-inoil type emulsion for treating a disease of the eye. United States patent US9, 107, 822. 2015.

[14] 14. Bachu RD, Stepanski M, Alzhrani RM, Jung R, Boddu SH. Development andevaluation of a novel microemulsion of dexamethasone and tobramycin fortopical ocular administration. J Ocul Pharmacol Ther;

2018. doi:http://doi.org/10.1089/jop.2017.0082. [Epub ahead of print].

[15] Ashara KC, Shah KV. Emulsion of chloramphenicol: an overwhelming approachfor ocular delivery. Folia Med 2017; 59:23-30.

[16] Gomes GV, Sola MR, Marostegan LF, Jange CG, Cazado $\mathrm{CP}$, Pinheiro AC, et al.Physico-chemical stability and in vitro digestibility of beta-carotene-loadedlipid nanoparticles of cupuacu butter (Theobroma grandiflorum) produced bythe phase inversion temperature (PIT) method. J Food Eng 2017;192:93102 
International Journal of Trend in Scientific Research and Development (IJTSRD) @ www.ijtsrd.com eISSN: 2456-6470

[17] Sharma AK, Garg T, Goyal AK, Rath G. Role of microemuslsions in advanced drug delivery. Artif Cells Nanomed Biotechnol 2016; 44:1177-85.

[18] Jain P, Rahi P, Pandey V, Asati S, Soni V. Nanostructure lipid carriers: a modishcontrivance to overcome the ultraviolet effects. EJBAS 2017; 4:89-100.

[19] Bansal D, Yadav K, Pandey V, Ganeshpurkar A, Agnihotri A, Dubey N. Lactobionic acid coupled liposomes: an innovative strategy for targetinghepatocellular carcinoma. Drug Deliv 2016; 23:140-6.

[20] Moustafa MA, Elnaggar YS, El-Refaie WM, Abdallah OY. Hyalugel-integratedliposomes as a novel ocular nanosized delivery system of fluconazole withpromising prolonged effect. Int J Pharm 2017; 534:14-24.
[21] Chetoni P, Monti D, Tampucci S, Matteoli B, CeccheriniNelli L, Subissi A, et al. Liposomes as a potential ocular delivery system of distamycin A. Int J Pharm2015;492:120-6.

[22] Dong Y, Dong P, Huang D, Mei L, Xia Y, Wang Z, et al. Fabrication andcharacterization of silk fibroin-coated liposomes for ocular drug delivery. Eur JPharm Biopharm 2015; 91:82-90.

[23] Chen Y, Tuo J, HuangH, Liu D, You X, Mai J, et al. Optimized mixed oils remarkablyreduce the amount of surfactants in microemulsions without affecting oral bioavailability of ibuprofen by simultaneously enlarging microemulsion areasand enhancing drug solubility. Int J Pharm 2015; 487:17-24.

[24] Mitra AK, ophthalmic drug delivery, In; Tyle P, editor drug delivery devices, new York, Marcel dekker;1998. 